\title{
High-Pressure Hydrogen Sulfide from First Principles: A Strongly Anharmonic Phonon-Mediated Superconductor
}

\author{
Ion Errea, ${ }^{1,2}$ Matteo Calandra, ${ }^{3, *}$ Chris J. Pickard, ${ }^{4}$ Joseph Nelson, ${ }^{5}$ Richard J. Needs, ${ }^{5}$ Yinwei Li, ${ }^{6}$ Hanyu Liu, \\ Yunwei Zhang, ${ }^{8}$ Yanming $\mathrm{Ma}^{8}{ }^{8}$ and Francesco Mauri ${ }^{3}$ \\ ${ }^{1}$ Donostia International Physics Center (DIPC), Manuel de Lardizabal Pasealekua 4, \\ 20018 Donostia-San Sebastián, Basque Country, Spain \\ ${ }^{2} I K E R B A S Q U E$, Basque Foundation for Science, Bilbao, Spain \\ ${ }^{3} I M P M C$, UMR CNRS 7590, Sorbonne Universités-UPMC University Paris 06, MNHN, IRD, 4 Place Jussieu, F-75005 Paris, France \\ ${ }^{4}$ Department of Physics \& Astronomy, University College London, Gower Street, London WC1E 6BT, United Kingdom \\ ${ }^{5}$ Theory of Condensed Matter Group, Cavendish Laboratory, J.J. Thomson Avenue, Cambridge CB3 OHE, United Kingdom \\ ${ }^{6}$ School of Physics and Electronic Engineering, Jiangsu Normal University, Xиzhou 221116, People's Republic of China \\ ${ }^{7}$ Department of Physics and Engineering Physics, University of Saskatchewan, Saskatchewan S7N 5E2, Canada \\ ${ }^{8}$ State Key Laboratory of Superhard Materials, Jilin University, Changchun 130012, People's Republic of China
}

(Received 10 February 2015; published 16 April 2015)

We use first-principles calculations to study structural, vibrational, and superconducting properties of $\mathrm{H}_{2} \mathrm{~S}$ at pressures $P \geq 200 \mathrm{GPa}$. The inclusion of zero-point energy leads to two different possible dissociations of $\mathrm{H}_{2} \mathrm{~S}$, namely $3 \mathrm{H}_{2} \mathrm{~S} \rightarrow 2 \mathrm{H}_{3} \mathrm{~S}+\mathrm{S}$ and $5 \mathrm{H}_{2} \mathrm{~S} \rightarrow 3 \mathrm{H}_{3} \mathrm{~S}+\mathrm{HS}_{2}$, where both $\mathrm{H}_{3} \mathrm{~S}$ and $\mathrm{HS}_{2}$ are metallic. For $\mathrm{H}_{3} \mathrm{~S}$, we perform nonperturbative calculations of anharmonic effects within the self-consistent harmonic approximation and show that the harmonic approximation strongly overestimates the electronphonon interaction ( $\lambda \approx 2.64$ at $200 \mathrm{GPa}$ ) and $T_{c}$. Anharmonicity hardens $\mathrm{H}-\mathrm{S}$ bond-stretching modes and softens $\mathrm{H}-\mathrm{S}$ bond-bending modes. As a result, the electron-phonon coupling is suppressed by $30 \%$ $(\lambda \approx 1.84$ at $200 \mathrm{GPa})$. Moreover, while at the harmonic level $T_{c}$ decreases with increasing pressure, the inclusion of anharmonicity leads to a $T_{c}$ that is almost independent of pressure. High-pressure hydrogen sulfide is a strongly anharmonic superconductor.

Cuprates [1] have for many years held the world record for the highest superconducting critical temperature $\left(T_{c}=133 \mathrm{~K}\right)$ [2]. However, despite almost 30 years of intensive research, the physical mechanism responsible for such a high $T_{c}$ is still elusive, although the general consensus is that it is highly nonconventional. The measurements by Drozdov et al. [3] which suggest a $190 \mathrm{~K}$ superconducting phase in hydrogen sulfide under high pressure (200 GPa), could break the cuprates record, if confirmed.

The claim that hydrogen at high pressure could be superconducting is not new [4] and it was recently supported by first-principles calculations based on the harmonic approximation applied to dense hydrogen [5-8] and several hydrides [9-15]. More recently, two theoretical papers predicted the occurrence of high- $T_{c}$ superconductivity in high-pressure sulfur hydrides $[16,17]$. However, as shown in Refs. [18,19], anharmonicity can be crucial in these systems. For example, in $\mathrm{PdH}$, the electron-phonon coupling $\lambda$ parameter is found to be 1.55 at the harmonic level, while a proper inclusion of anharmonic effects leads to $\lambda=0.40$ [18], in better agreement with experiments. Thus, in hydrogen-based compounds, the phonon spectra are strongly affected by anharmonic effects.

Given the sensitivity of superconductivity to the physical and electronic structures, it is extremely important to identify the correct crystal structures (see, for example, the early discussion of superconductivity in silane in Ref. [15], and one of the first applications of first-principles structure prediction in Ref. [20]). Several first-principles calculations [16,17,21-23] suggested that decomposition of the $\mathrm{H}_{2} \mathrm{~S}$ sample occurs within the diamond-anvil cell at high pressures. The high- $T_{c}$ superconducting material is therefore very unlikely to be $\mathrm{H}_{2} \mathrm{~S}$, while $\mathrm{H}_{3} \mathrm{~S}$ is the obvious candidate for the H-rich decomposition product.

Here we study the structural, vibrational, and superconducting properties of $\mathrm{H}_{2} \mathrm{~S}$ above $200 \mathrm{GPa}$, where the highest $T_{c}$ occurs. We show that the inclusion of zero-point motion in the convex hull at 200 and $250 \mathrm{GPa}$ stabilizes two metallic structures, $\mathrm{H}_{3} \mathrm{~S}$ and $\mathrm{HS}_{2}$. Finally, we show that, contrary to suggestions in previous work $[16,21]$, the harmonic approximation does not explain the measured $T_{c}$ in $\mathrm{H}_{3} \mathrm{~S}$, and the inclusion of anharmonic effects is crucial.

As decomposition of $\mathrm{H}_{2} \mathrm{~S}$ has been demonstrated in the experiments of Ref. [3], it is crucial to develop an understanding of the different $\mathrm{H}-\mathrm{S}$ compounds that might be stable in the pressure range of interest. We therefore perform a search over $44 \mathrm{H}: \mathrm{S}$ stoichiometries, determining the stoichiometries at which stable structures exist, and the associated crystal structures. These searches were performed with the $a b$ initio random structure searching 


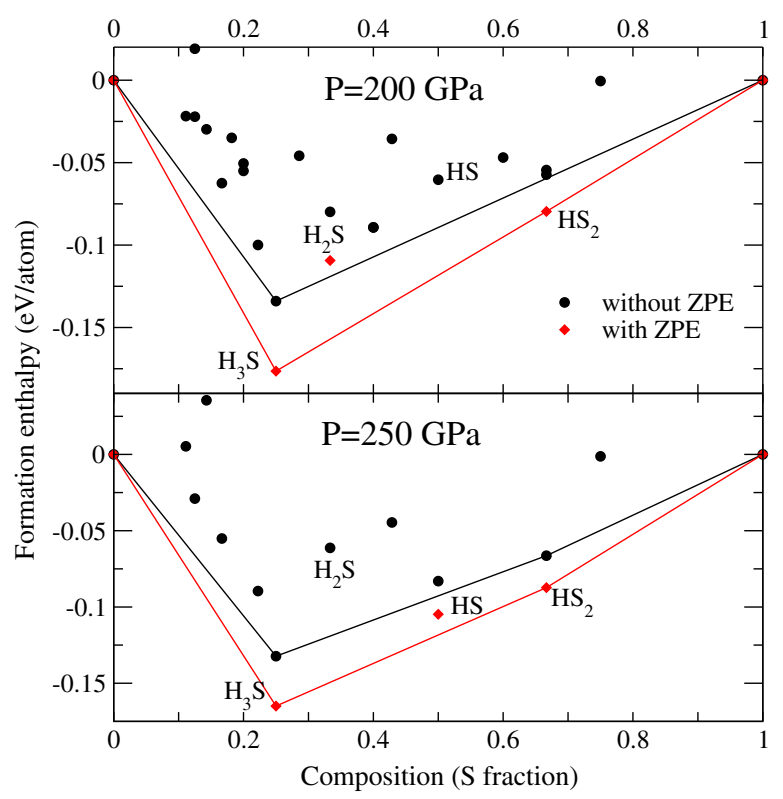

FIG. 1 (color online). Results of structure searching at 200 and $250 \mathrm{GPa}$. Convex hulls are shown as continuous lines, with and without the inclusion of zero-point energy (ZPE).

(AIRSS) method [20,24] and the CASTEP code [25], and the CALYPSO particle-swarm optimization method [26,27] and the VASP code $[28,29]$. More information about the searches is provided in the Supplemental Material [30].

The results of the structure searching are shown in Fig. 1. At $200 \mathrm{GPa}$, without zero-point energy (ZPE), the only energetically allowed decomposition is $3 \mathrm{H}_{2} \mathrm{~S} \rightarrow 2 \mathrm{H}_{3} \mathrm{~S}+\mathrm{S}$, in agreement with previous calculations $[16,21,22,34] . \mathrm{H}_{3} \mathrm{~S}$ crystallizes in the space group $\operatorname{Im} \overline{3} m$, as shown in Ref. [16]. When ZPE is included, a second decomposition becomes possible at $200 \mathrm{GPa}$, namely $5 \mathrm{H}_{2} \mathrm{~S} \rightarrow 3 \mathrm{H}_{3} \mathrm{~S}+\mathrm{HS}_{2}$, where $\mathrm{HS}_{2}$ crystallizes in a structure of space group $C 2 / c$ with 12 atoms/cell. At $250 \mathrm{GPa}$ and above, the latter decomposition is allowed even without $\mathrm{ZPE}$, and the $C 2 / c \mathrm{HS}_{2}$ structure undergoes a phase transition to a more stable $C 2 / m$ structure with 6 atoms/cell. Each of the $\mathrm{HS}_{2}$ structures is metallic. Finally, at $300 \mathrm{GPa}$, a metallic HS phase with $C 2 / m$ space group becomes stable [30]. Detailed information on the crystal structures is provided in the Supplemental Material [30].

Having determined the most stable crystal structures at high pressure, we turn to the study of vibrational properties $[35,36]$. As summarized in Table I, the superconducting $T_{c}$ values obtained within the harmonic approximation for the $C 2 / c \mathrm{HS}_{2}, C 2 / m \mathrm{HS}_{2}$, and $C 2 / m$ HS phases are in the range of 15 to $35 \mathrm{~K}$ (see Supplemental Material [30] for phonon spectra and details of the calculations), far from the observed extraordinary values. The measured $T_{c}$ 's could not have occurred in any of these phases. Thus, we focus on the $\operatorname{Im} \overline{3} m \mathrm{H}_{3} \mathrm{~S}$ structure. In this structure, each $\mathrm{H}$ atom is twofold coordinated and has 6 neighbors, 2 of which are $\mathrm{S}$ atoms while the other 4 are $\mathrm{H}$ atoms. The $\mathrm{H}$ vibrations can
TABLE I. Calculated $\lambda, \omega_{\log }$, and $T_{c}$ values in the harmonic approximation for $C 2 / c \mathrm{HS}_{2}, C 2 / m \mathrm{HS}_{2}$, and $C 2 / m \mathrm{HS} . T_{c}$ is estimated using the McMillan equation.

\begin{tabular}{lccccc}
\hline \hline Phase & $P(\mathrm{GPa})$ & $\lambda$ & \multicolumn{2}{c}{$\omega_{\log }(\mathrm{meV})$} & $T_{c}^{\mu^{*}=0.10}(\mathrm{~K}) T_{c}^{\mu^{*}=0.16}(\mathrm{~K})$ \\
\hline$C 2 / c \mathrm{HS}_{2}$ & 200 & 0.86 & 56.7 & 35.3 & 23.4 \\
$C 2 / m \mathrm{HS}_{2}$ & 250 & 0.75 & 53.8 & 25.1 & 14.9 \\
$C 2 / m \mathrm{HS}$ & 300 & 0.78 & 74.3 & 38.0 & 23.4 \\
\hline \hline
\end{tabular}

then be decomposed into $\mathrm{H}-\mathrm{S}$ bond-stretching modes $\left(\mathrm{H}_{\|}\right)$, in which an $\mathrm{H}$ atom moves toward one of the two $\mathrm{S}$ atoms, and bond-bending modes $\left(\mathrm{H}_{\perp}\right)$, in which one $\mathrm{H}$ atom moves in the direction perpendicular to the $\mathrm{H}-\mathrm{S}$ bond (see
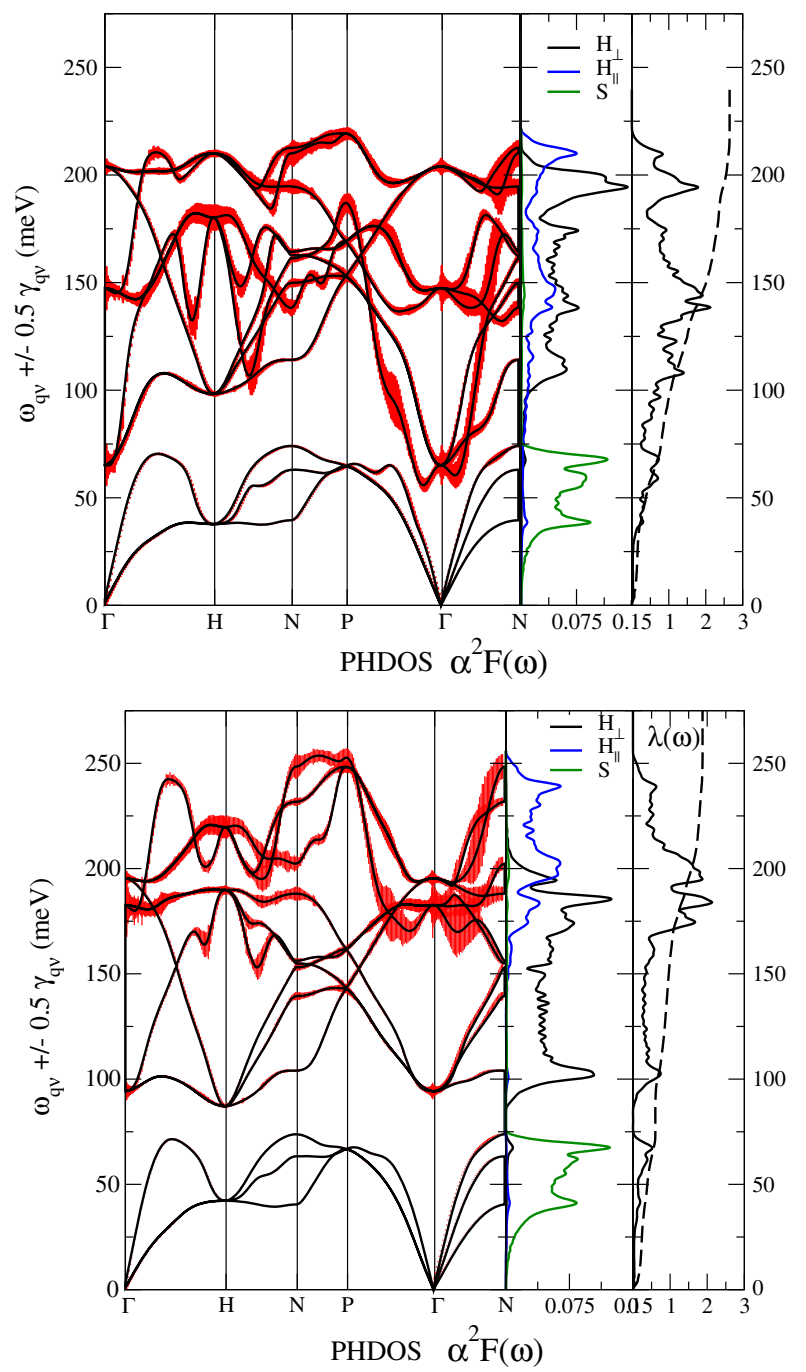

FIG. 2 (color online). Phonon dispersion, phonon density of states projected onto selected atoms and directions, and the Eliashberg function of $\mathrm{H}_{3} \mathrm{~S}$ in the harmonic approximation (top) and with the inclusion of anharmonic effects (bottom) for $\mathrm{H}_{3} \mathrm{~S}$ at $200 \mathrm{GPa} . \mathrm{H}_{\perp}$ and $\mathrm{H}_{\|}$label displacements of an $\mathrm{H}$ atom in the directions perpendicular or parallel to a $\mathrm{H}-\mathrm{S}$ bond. The magnitude of the phonon linewidth is indicated by the size of the red error bars. 
Fig. 1 in the Supplemental Material [30]). The harmonic phonon spectrum of $\mathrm{H}_{3} \mathrm{~S}$ at $200 \mathrm{GPa}$ [39] is shown in Fig. 2 and overall shows a clear separation into $\mathrm{H}$ modes at high energy and $\mathrm{S}$ modes below $75 \mathrm{meV}$. To gain more insight, we use Wannier interpolation [40,41] of the electronphonon matrix elements and evaluate the electron-phonon contribution to the phonon linewidth as [42]

$$
\gamma_{\mathbf{q} \nu}=\frac{4 \pi \omega_{\mathbf{q} \nu}}{N_{k}} \sum_{\mathbf{k}, n, m}\left|g_{n m}^{\nu}(\mathbf{k}, \mathbf{k}+\mathbf{q})\right|^{2} \delta\left(\varepsilon_{\mathbf{k} n}\right) \delta\left(\varepsilon_{\mathbf{k}+\mathbf{q} m}\right) .
$$

Here, $\omega_{\mathbf{q} \nu}$ are the phonon frequencies, $N_{k}$ the number of electron-momentum points in the grid, $g_{n m}^{\nu}(\mathbf{k}, \mathbf{k}+\mathbf{q})=$ $\left\langle\mathbf{k} n\left|\delta V_{\mathrm{KS}} / \delta u_{\mathbf{q} \nu}\right| \mathbf{k}+\mathbf{q} m\right\rangle$ is the electron-phonon matrix element, $V_{\mathrm{KS}}$ is the Kohn-Sham potential, and $u_{\mathbf{q} \nu}$ is a phonon displacement. The Kohn-Sham energy and eigenfunctions are labeled $\varepsilon_{\mathbf{k} n}$ and $|\mathbf{k} n\rangle$. The electron-phonon coupling at a given phonon-momentum $\mathbf{q}$ for a phonon mode $\nu$ can be obtained [42] from the phonon linewidth as $\lambda_{\mathbf{q} \nu}=\left\{\gamma_{\mathbf{q} \nu} /\left[2 \pi \omega_{\mathbf{q} \nu}^{2} N(0)\right]\right\}$.

As shown in Fig. 2, at the harmonic level, the phonon linewidths of the $\mathrm{H}$ vibrations are fairly uniform throughout the spectrum. The contribution of each mode to the average electron-phonon interaction, $\lambda=\sum_{\nu \mathbf{q}} \lambda_{\mathbf{q} \nu} / N_{q}$, can be obtained from the isotropic Eliashberg function,

$$
\alpha^{2} F(\omega)=\frac{1}{2 N_{q}} \sum_{\mathbf{q} \nu} \lambda_{\mathbf{q} \nu} \omega_{\mathbf{q} \nu} \delta\left(\omega-\omega_{\mathbf{q} \nu}\right),
$$

where $N_{q}$ is the number of phonon-momentum points in the grid. $\lambda(\omega)=2 \int_{0}^{\omega}\left[\alpha^{2} F\left(\omega^{\prime}\right) / \omega^{\prime}\right] d \omega^{\prime}$ and then $\lambda=\lambda(\infty)$. We find $\lambda=2.64$ (see Table II), which is larger than that obtained in Refs. [16,21] with a much coarser sampling of the BZ, but consistent with the result in Ref. [22]. This huge value of $\lambda$ comprises substantial contributions from many $\mathrm{H}$ vibrational modes. The situation is therefore very different from $\mathrm{MgB}_{2}$ in which a single mode dominates $\lambda$.

Given the low mass of $\mathrm{H}$ and the consequent large phonon displacements, we investigate the occurrence of anharmonic effects using the stochastic self-consistent harmonic approximation (SSCHA) developed by some of us $[18,19,43]$. As shown in Fig. 2 (bottom), the anharmonic correction leads to nontrivial changes in the harmonic spectrum. While it is very clear that all $\mathrm{H}$ bond-stretching modes are hardened, the effect on $\mathrm{H}$ bond-bending modes is less straightforward. By computing the average phonon frequency as of $\mathrm{H}_{\|}$and of $\mathrm{H}_{\perp}$ modes, we find that $\bar{\omega}_{\|}^{\text {har }} \approx 158.1 \mathrm{meV}$ and $\bar{\omega}_{\|}^{\text {anh }} \approx 203.3 \mathrm{meV}$, while for bond-bending modes $\bar{\omega}_{\perp}^{\text {har }} \approx 157.0 \mathrm{meV}$ and $\bar{\omega}_{\perp}^{\mathrm{anh}} \approx 147.9 \mathrm{meV}$. Thus bond-stretching modes are hardened, while bond-bending modes are softened.

It is important to remark that the large and most dispersive mode along $P \Gamma$ is strongly hardened at the anharmonic level and undergoes a nontrivial change in polarization, as can be seen from the large effect of anharmonicity on the phonon linewidth $\gamma_{\mathbf{q} \nu}$ in Fig. 2. It is worthwhile recalling that the phonon linewidth depends on the phonon eigenvector but not on the phonon energy. This effect demonstrates the need to calculate not only the phonon frequencies at the anharmonic level, but also the phonon polarizations.

The anharmonic electron-phonon interaction is $\lambda=1.84$, which is $30 \%$ smaller than the harmonic result. This reduction is mostly explained by the hardening of the $\mathrm{H}_{\|}$modes. In contrast to the harmonic case, which shows uniform coupling over all modes, the anharmonic Eliashberg function has two main peaks, a broad peak in the $40-75 \mathrm{meV}$ region, and a second one in the 175$200 \mathrm{meV}$ region. Their contributions to $\lambda$ are 0.59 and 0.77 , respectively, accounting for $\approx 73 \%$ of the total $\lambda$. We note, however, that the logarithmic average of the phonon frequencies $\omega_{\log }$ is only weakly enhanced by anharmonicity (see Table II).

The superconducting critical temperature can be obtained either from the McMillan equation or the isotropic Migdal-Eliashberg approach. However, it is well known [44] that the use of the McMillan equation for such values of $\lambda$ leads to a substantial underestimation of $T_{c}$. We solved the isotropic Eliashberg equations (see the Supplemental Material [30]) and found, contrary to claims in previous publications $[16,21]$, that calculations based on the harmonic phonon spectrum do not explain the measured $T_{c}$ as, even using large values of $\mu^{*}[45,46], T_{c}$ is substantially overestimated (i.e., $T_{c}=250 \mathrm{~K}$ for $\mu^{*}=0.16$ [30]). When the anharmonic phonon spectrum and electronphonon coupling are used, the Migdal-Eliashberg equations account for the experimental $T_{c}$ when the value $\mu^{*}=0.16$

TABLE II. Electron-phonon interaction and logarithmic averages of phonon frequencies, with and without anharmonic effects. The $T_{c}$ 's are calculated using the isotropic Migdal-Eliashberg equations $\left(T_{c}^{\mathrm{ME}}\right)$. A value of $\mu^{*}=0.16$ is used. Data for $T_{c}$ calculated with the McMillan equation are provided in the Supplemental Material [30]. Frequencies are in $\mathrm{meV}$ and $T_{c}$ 's are in $\mathrm{K}$.

\begin{tabular}{llclcccc}
\hline \hline Compound & $\lambda^{\text {har }}$ & $\omega_{\log }^{\text {har }}$ & $\lambda^{\text {anh }}$ & $\omega_{\log }^{\text {anh }}$ & $T_{c}^{\text {ME,har }}$ & $T_{c}^{\text {ME,anh }}$ & $T_{c}$ (expt.) \\
\hline $\mathrm{H}_{3} \mathrm{~S}(200 \mathrm{GPa})$ & 2.64 & 90.4 & 1.84 & 92.86 & 250 & 194.0 & 190 \\
$\mathrm{H}_{3} \mathrm{~S}(250 \mathrm{GPa})$ & 1.96 & 109.1 & 1.71 & 101.3 & 226 & 190 & 150 \\
$\mathrm{D}_{3} \mathrm{~S}(200 \mathrm{GPa})$ & 2.64 & 68.5 & 1.87 & 73.3 & 183 & 152.0 & 90 \\
\hline \hline
\end{tabular}


is used, as shown in Table II. The superconducting gap at zero temperature is $\Delta \approx 36.5 \mathrm{meV}$. By using the smaller value of $\mu^{*}=0.1$, we obtain $T_{c}=222 \mathrm{~K}$ and $\Delta \approx 43 \mathrm{meV}$.

Interestingly, the large anharmonic effects lead to very different variations in $T_{c}$ with pressure. By performing the harmonic electron-phonon calculation for the $\operatorname{Im} \overline{3} m$ structure at $250 \mathrm{GPa}$ and using the Migdal-Eliashberg equations with $\mu^{*}=0.16$ we found $T_{c}=226 \mathrm{~K}$, decreasing with increasing pressure. However, at the anharmonic level, we find $T_{c}=190 \mathrm{~K}$, essentially independent of pressure in the region 200-250 GPa.

Finally, we consider the extent to which the occurrence of large anharmonic effects can explain the isotope shift in $\mathrm{D}_{2} \mathrm{~S}$. At $164 \mathrm{GPa}, T_{c}\left(\mathrm{D}_{2} \mathrm{~S}\right)=90 \mathrm{~K}$, leading to an isotope coefficient $\alpha \approx 1.07$, which is substantially enhanced from the canonical BCS value of $\alpha \approx 0.5$. Assuming a similar decomposition of $\mathrm{D}_{2} \mathrm{~S}$ into $\mathrm{D}_{3} \mathrm{~S}$ and $\mathrm{S}$ at high pressures, we calculate the anharmonic phonon spectrum (see the Supplemental Material [30]) and electron-phonon coupling in $\mathrm{D}_{3} \mathrm{~S}$ at $200 \mathrm{GPa}$. We find at the anharmonic level that the electron-phonon coupling is essentially unaffected, while $\omega_{\log }$ is softened from $92.9 \mathrm{meV}$ to $73.3 \mathrm{meV}$, leading to an isotope coefficient of $\alpha=0.35$, which is strongly reduced from the BCS value. Thus, contrary to the claim made in Ref. [47], anharmonicity reduces $\alpha$. As long as the same crystal structure for $\mathrm{H}_{3} \mathrm{~S}$ and $\mathrm{D}_{3} \mathrm{~S}$ is considered, as predicted by our calculation, the theoretical isotope coefficient remains inconsistent with the value of $\alpha \approx 1.07$ found in experiments. This disagreement could be reconciled if in experiment kinetics stabilize different structures for hydrides and deuterides at a given pressure. More experimental data are necessary to clarify this issue.

We have studied the structural, vibrational, and superconducting properties of high-pressure $\mathrm{H}_{3} \mathrm{~S}$. We have included zero-point motion when comparing the stabilities of different H-S phases, which has been neglected in other publications so far. This is important because zero-point motion stabilizes a new phase at $P \geq 200 \mathrm{GPa}$. In particular, we found that decomposition of $\mathrm{HS}_{2}$ into metallic phases can occur following two main paths, namely $3 \mathrm{H}_{2} \mathrm{~S} \rightarrow 2 \mathrm{H}_{3} \mathrm{~S}+\mathrm{S}$ and $5 \mathrm{H}_{2} \mathrm{~S} \rightarrow 3 \mathrm{H}_{3} \mathrm{~S}+\mathrm{HS}_{2}$. We have performed a detailed study of the vibrational properties of high-pressure $\mathrm{H}_{3} \mathrm{~S}$ and $\mathrm{D}_{3} \mathrm{~S}$, finding that the phonon spectra are strongly affected by anharmonic effects. Anharmonicity hardens $\mathrm{H}-\mathrm{S}$ bond-stretching modes and softens $\mathrm{H}-\mathrm{S}$ bond-bending modes. Moreover, anharmonicity leads to a reduction in the electron-phonon coupling by $\approx 30 \%$ and to an approximately constant $T_{c}$ in the range 200-250 GPa. Our work demonstrates that the superconducting properties of high-pressure $\mathrm{H}_{3} \mathrm{~S}$ can only be properly described by including both nuclear quantum effects and anharmonicity.

We acknowledge discussions with I. I. Mazin and support from the Agence Nationale de la Recherche, Grant
No. ANR-13-IS10-0003-01. Computer facilities were provided by PRACE, CINES, CCRT, and IDRIS. I.E. acknowledges financial support from the Department of Education, Language Policy, and Culture of the Basque Government (Grant No. BFI-2011-65) and the Spanish Ministry of Economy and Competitiveness (FIS201348286-C2-2-P). C. J. P. and R. J. N. thank EPSRC (UK) for financial support. J. R. N. acknowledges financial support from the Cambridge Commonwealth Trust. Y. Li thanks the National Natural Science Foundation of China for support under Grants No. 11204111 and No. 11404148. Y. Zhang and Y. Ma thank the Natural Science Foundation of China for support under Grant No. 11274136 and the 2012 Changjiang Scholars Program of China.

*matteo.calandra@upmc.fr

[1] J. G. Bednorz and K. A. Mueller, Z. Phys. B 64, 189 (1986).

[2] A. Schilling, M. Cantoni, J. D. Guo, and H. R. Ott, Nature (London) 363, 56 (1993).

[3] A. P. Drozdov, M. I. Eremets, and I. A. Troyan, arXiv:1412.0460.

[4] N. W. Ashcroft, Phys. Rev. Lett. 21, 1748 (1968).

[5] L. Zhang, Y. Niu, Q. Li, T. Cui, Y. Wang, Y. Ma, Z. He, and G. Zou, Solid State Commun. 141, 610 (2007).

[6] P. Cudazzo, G. Profeta, A. Sanna, A. Floris, A. Continenza, S. Massidda, and E. K. U. Gross, Phys. Rev. Lett. 100, 257001 (2008).

[7] P. Cudazzo, G. Profeta, A. Sanna, A. Floris, A. Continenza, S. Massidda, and E. K. U. Gross, Phys. Rev. B 81, 134505 (2010).

[8] P. Cudazzo, G. Profeta, A. Sanna, A. Floris, A. Continenza, S. Massidda, and E. K. U. Gross, Phys. Rev. B 81, 134506 (2010).

[9] D. Y. Kim, R. H. Scheicher, C. J. Pickard, R. J. Needs, and R. Ahuja, Phys. Rev. Lett. 107, 117002 (2011).

[10] T. Scheler, O. Degtyareva, M. Marques, C. L. Guillaume, J. E. Proctor, S. Evans, and E. Gregoryanz, Phys. Rev. B 83, 214106 (2011).

[11] X.-F. Zhou, A. R. Oganov, X. Dong, L. Zhang, Y. Tian, and H.-T. Wang, Phys. Rev. B 84, 054543 (2011).

[12] D. Y. Kim, R. H. Scheicher, H.-k. Mao, T. W. Kang, and R. Ahuja, Proc. Natl. Acad. Sci. U.S.A. 107, 2793 (2010).

[13] G. Gao, A. R. Oganov, P. Li, Z. Li, H. Wang, T. Cui, Y. Ma, A. Bergara, A. O. Lyakhov, T. Iitaka, and G. Zou, Proc. Natl. Acad. Sci. U.S.A. 107, 1317 (2010).

[14] G. Gao, A. R. Oganov, A. Bergara, M. Martinez-Canales, T. Cui, T. Iitaka, Y. Ma, and G. Zou, Phys. Rev. Lett. 101, 107002 (2008).

[15] J. Feng, W. Grochala, T. Jaron, R. Hoffmann, A. Bergara, and N. W. Ashcroft, Phys. Rev. Lett. 96, 017006 (2006).

[16] D. Duan, Y. Liu, F. Tian, D. Li, X.-a. Huang, Z. Zhao, H. Yu, B. Liu, W. Tian, and T. Cui, Sci. Rep. 4, 6968 (2014).

[17] Y. Li, J. Hao, H. Liu, Y. Li, and Y. Ma, J. Chem. Phys. 140, 174712 (2014).

[18] I. Errea, M. Calandra, and F. Mauri, Phys. Rev. Lett. 111, 177002 (2013). 
[19] I. Errea, M. Calandra, and F. Mauri, Phys. Rev. B 89, 064302 (2014).

[20] C. J. Pickard and R. J. Needs, Phys. Rev. Lett. 97, 045504 (2006).

[21] J. A. Flores-Livas, A. Sanna, and E. K. U. Gross, arXiv:1501.06336.

[22] R. Akashi, M. Kawamura, S. Tsuneyuki, Y. Nomura, and R. Arita, arXiv:1502.00936.

[23] D. Duan, X. Huang, F. Tian, D. Li, Hongyu, Yu, Y. Liu, Y. Ma, B. Liu, and T. Cui, arXiv:1501.01784.

[24] C. J. Pickard and R. J. Needs, J. Phys. Condens. Matter 23, 053201 (2011).

[25] S. J. Clark, M. D. Segall, C. J. Pickard, P. J. Hasnip, M. I. J. Probert, K. Refson, and M. C. Payne, Z. Kristallogr. 220, 567 (2005).

[26] Y. Wang, J. Lv, L. Zhu, and Y. Ma, Comput. Phys. Commun. 183, 2063 (2012).

[27] Y. Wang, J. Lv, L. Zhu, and Y. Ma, Phys. Rev. B 82, 094116 (2010).

[28] G. Kresse and J. Furthmüller, Comput. Mater. Sci. 6, 15 (1996).

[29] G. Kresse and J. Furthmüller, Phys. Rev. B 54, 11169 (1996).

[30] See Supplemental Material at http://link.aps.org/ supplemental/10.1103/PhysRevLett.114.157004 for convex hull of $\mathrm{H}_{x} \mathrm{~S}_{y}$ at $300 \mathrm{GPa}$, the effect of the zero-point energy on the convex hull at $200 \mathrm{GPa}$, crystal structures, electronic structure of $\mathrm{H}_{3} \mathrm{~S}$, vibrational properties of $\mathrm{D}_{3} \mathrm{~S}$ at $200 \mathrm{GPa}$ and $\mathrm{H}_{3} \mathrm{~S}$ at $250 \mathrm{GPa}$, solution of Migdal-Eliashberg equations, the effects of the vibrational zero-point energy on pressure, electronic and vibrational properties of $\mathrm{HS}_{2}$ and HS phases. Supplemental Material includes Refs. [31-33].

[31] A. Togo, F. Oba, and I. Tanaka, Phys. Rev. B 78, 134106 (2008).

[32] C. J. Pickard and R. J. Needs, Nat. Phys. 3, 473 (2007).

[33] O. Degtyareva, E. Gregoryanz, M. Somayazulu, H.-k. Mao, and R. J. Hemley, Phys. Rev. B 71, 214104 (2005).

[34] N. Bernstein, C. S. Hellberg, M. D. Johannes, I. I. Mazin, and M. J. Mehl, Phys. Rev. B 91, 060511 (2015).

[35] P. Giannozzi et al., J. Phys. Condens. Matter 21, 395502 (2009).

[36] Our superconductivity results were obtained from firstprinciples DFT linear-response calculations as implemented in the QUANTUM-ESPRESSO [35] package. We used ultrasoft [37] pseudopotentials, a generalized gradient approximation [38], a plane-wave cutoff energy of $60 \mathrm{Ry}$ on the kinetic energy and $600 \mathrm{Ry}$ on the charge density. The charge density and dynamical matrices were calculated using a $32^{3}$ Monkhorst-Pack shifted electron-momentum grid and a Hermitian-Gaussian smearing of 0.03 Ry. The average electron-phonon coupling was obtained using Wannier interpolation [40]. We used a $32^{3}$ electron-momentum grid randomly shifted from the origin and a $32^{3}$ Monkhorst-Pack shifted phonon-momentum grid with a smearing of $0.03 \mathrm{eV}$. The phonon linewidth at a given phonon momentum was calculated using a $50 \times 50 \times 50$ electron-momentum mesh (randomly shifted from the origin).

[37] D. Vanderbilt, Phys. Rev. B 41, 7892 (1990).

[38] J. P. Perdew, K. Burke, and M. Ernzerhof, Phys. Rev. Lett. 77, 3865 (1996).

[39] The pressure computed here is without inclusion of ZPE. Neglecting ZPE underestimates the pressure of $\approx 12 \mathrm{GPa}$ for $\mathrm{H}_{3} \mathrm{~S}$ and $6 \mathrm{GPa}$ for $\mathrm{D}_{3} \mathrm{~S}$ for a volume of 89.9822 (a.u.) ${ }^{3}$. See the Supplemental Material [30] for more details.

[40] M. Calandra, G. Profeta, and F. Mauri, Phys. Rev. B 82, 165111 (2010).

[41] F. Giustino, J. R. Yates, I. Souza, M. L. Cohen, and G. Louie, Phys. Rev. Lett. 98, 047005 (2007).

[42] P. B. Allen, Phys. Rev. B 6, 2577 (1972); P. B. Allen and R. Silberglitt, Phys. Rev. B 9, 4733 (1974).

[43] The SSCHA calculations were performed using a $3 \times 3 \times 3$ supercell for both $\mathrm{H}_{3} \mathrm{~S}$ and $\mathrm{D}_{3} \mathrm{~S}$ at $0 \mathrm{~K}$, yielding dynamical matrices on a commensurate $3 \times 3 \times 3 q$-point grid. An additional calculation at $200 \mathrm{~K}$ confirmed that temperatures within the range of the predicted superconductivity do not affect the phonon spectra. The difference between the harmonic and anharmonic dynamical matrices in the $3 \times 3 \times 3$ phonon-momentum grid was interpolated to a $6 \times 6 \times 6$ grid. Adding the harmonic matrices to the result, the anharmonic dynamical matrices were obtained in a $6 \times 6 \times 6$ grid. These dynamical matrices were used for the anharmonic electron-phonon coupling calculation.

[44] P. B. Allen and R. C. Dynes, Phys. Rev. B 12, 905 (1975).

[45] P. Morel and P. W. Anderson, Phys. Rev. 125, 1263 (1962).

[46] N. N. Bogoliubov, V. V. Tolmachev, and D. V. Shirkov, A New Method in the Theory of Superconductivity (Consultants Bureau, New York, 1959).

[47] D. A. Papaconstantopoulos, B. M. Klein, M. J. Mehl, and W. E. Pickett, arXiv:1501.03950v1. 